\title{
Low-fidelity simulation of medical emergency and cardiac arrest responses in a suspected COVID-19 patient - an interim report
}

\author{
Authors: Rhys D Wenlock, ${ }^{A}$ Amy Arnold, ${ }^{A}$ Hiten Patel ${ }^{B}$ and David Kirtchuk ${ }^{B}$
}

\begin{abstract}
Background
COVID-19 poses many challenges to healthcare systems and workers. Responding to medical emergencies in patients with suspected COVID-19 will require new guidelines and protocols. Simulation can support their development.

Methods

We organised seven simulations involving patients with suspected COVID-19 for staff at Brighton and Sussex University Hospitals. Participants completed pre- and postsimulation questionnaires.

Results

Fifty-six staff participated and they reported being significantly less prepared to respond to an emergency in a patient with suspected COVID-19 than in one in whom it is not suspected. The simulations significantly improved the participants' confidence in responding to emergencies in patients with suspected COVID-19. Numerous challenges were identified along the themes of equipment, personnel, communication and procedures.

\section{Conclusions}

Low-fidelity simulation can provide relevant and timely information on how prepared health systems and their workforce are to respond to emergencies. We urge NHS trusts nationally to implement simulations to identify problems and develop effective solutions.
\end{abstract}

KEYWORDS: Infectious diseases, simulation, resuscitation, quality improvement, COVID-19

DOI: $10.7861 /$ clinmed.2020-0142

\section{Introduction}

Simulation is playing an increasing role in medical education globally. It provides the opportunity to assess not only clinical knowledge, but also the soft skills that are required to work

Authors: ${ }^{A}$ foundation year 1 doctor, Brighton and Sussex University Hospitals NHS Trust, Brighton, UK; ${ }^{\text {B }}$ Cardiology registrar, Brighton and Sussex University Hospitals NHS Trust, Brighton, UK effectively in emergency situations without compromising patient safety. There has been a large focus on high-fidelity simulation that acts to replicate the working environment as closely as possible, with the literature supporting its effectiveness as a teaching method. ${ }^{2,3}$ High-fidelity simulation is often undertaken in a simulation suite utilising video playback software and responsive mannequins. Less focus has been placed on low-fidelity simulation (which uses the minimal resources necessary to achieve its learning objectives). However, it can be used effectively to stress-test systems and to provide clinical staff with exposure to situations in their usual work context. ${ }^{4}$

The global COVID-19 pandemic is expected to challenge health systems universally, with the expected number of acutely unwell patients predicted to increase drastically. ${ }^{5}$ As a result of this, hospitals, staff and their systems will have to adapt to ever-changing conditions. This particularly relates to the use of personal protective equipment (PPE) and the approach to medical emergencies. Low-fidelity simulation not only has the potential to provide staff with more experience in these areas, but also allows the testing and improvement of protocols and guidance. ${ }^{6}$

At this NHS trust, a medical emergency team (MET) responds to emergencies across the hospital site. Depending on the nature of the emergency call placed, a multidisciplinary team responds and includes the medical registrar, several below-registrar grade junior doctors, the critical care outreach team (CCOT) and anaesthetists/ intensivists.

This study aims to pilot a low-fidelity COVID-19 MET response simulation to improve our understanding of the role that simulation can play in this global crisis, to offer a safe training environment to staff, and to identify problems with the current protocols while providing workable solutions.

\section{Methods}

We organised and ran seven simulations in barrier-nursed side rooms at Brighton and Sussex University Hospitals NHS Trust. The simulation team consisted of one patient (a junior doctor), two ward nurses (one acting as initiator), one ward doctor (FY1 grade), and the medical emergency / cardiac arrest team (a three- to fourperson team comprising a combination of FY1s, SHOs and SpRs). Scenarios were run as realistically as possible (eg no response until a simulated call was placed, donning and doffing of appropriate PPE, use of the resuscitation trolley, and simulation of arterial blood gases (ABG) / venepuncture / cannulation). 
To ensure that we had an adequate overview of the scenarios, two facilitators observed different aspects of the simulation. One provided observations and clinical findings to the participants, on request, from a pre-designed simulation template. The other was tasked with observing and providing feedback focused on the communication and procedural issues of running an emergency and arrest call through a closed door. The simulations were often observed by non-participating members of the ward team to maximise interprofessional learning. We asked all participants to complete an online pre- and post-simulation questionnaire and feedback form, hosted on Qualtrics, reviewing perceived preparedness for MET / cardiac arrest calls in patients with suspected or confirmed COVID-19 (supplementary materials).

\section{Scenario one}

> A 75-year-old male was admitted with a presumed diagnosis of decompensated heart failure, for which he required continuous positive airway pressure (CPAP); however, having also presented with fever and a cough, the COVID-19 pathway had been triggered. He was isolated in a side room, with contacts required to wear full PPE. The scenario begins with the nurse placing an emergency call as he becomes unresponsive and shows no signs of life.

\section{Scenario two}

$>$ We wanted to focus on how the team would deal with managing a MET call that did not require full aerosol-generating PPE but where the patient deteriorates, mid-simulation, into a cardiac arrest to monitor efficiency and time to don and doff PPE in a time-critical scenario. To ensure that we did not overload the participants, we used the same clinical background as scenario one. The scenario commences with an acutely unwell patient admitted with decompensated heart failure on nasal oxygen, but being barrier nursed as a query COVID-19 case. The nurse puts out an emergency call as he deteriorates, becoming hypoxic and tachypnoeic. The scenario follows an initial A-E assessment, with the team realising the need for CPAP. The patient then becomes unresponsive with no signs of life before CPAP can be set up. A cardiac arrest scenario follows.

Both scenarios were stopped by the faculty once a suitable end point had been reached (based upon the simulation template). In between each scenario, the faculty led the debrief focusing on procedural issues, as well as soft skills feedback to individual participants. These were documented and collated by the facilitators, and solutions were proposed where possible.

Data were imported and analysed in R Studio (v1.02.5033). Statistics were performed using a two-sample paired t-test.

\section{Results}

Fifty-six people participated in the simulations and completed the surveys. FY1s were the largest contingent with 22 of them participating in the simulations, followed by 16 nurses, $14 \mathrm{SHOs}$, three SpRs and one other. Of the 56 participants, 20\% ( $n=11)$ had been to a previous MET call in a patient with suspected COVID-19 and $5 \%(n=3)$ had been to a cardiac arrest in a patient with suspected COVID-19.

The majority of respondents $(87.5 \%, n=49)$ reported knowing where to find local trust guidelines, and the majority had reviewed

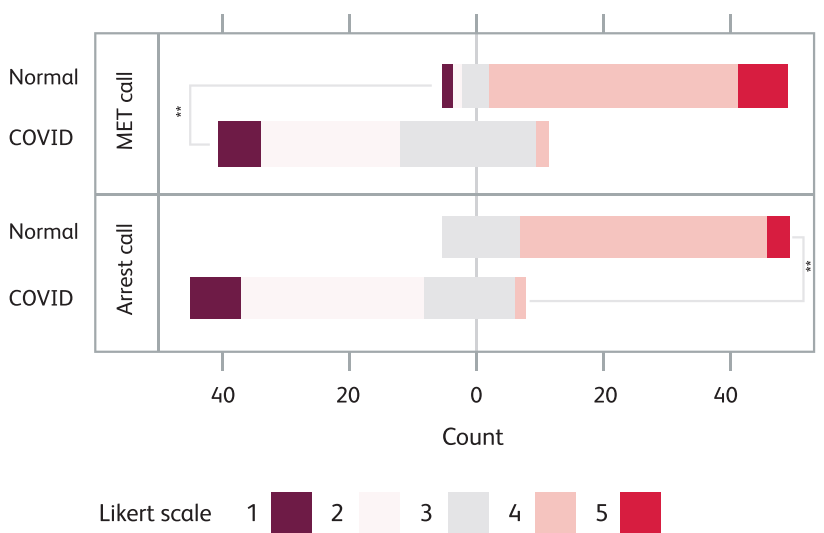

Fig 1. The responses on a Likert scale (1: completely unprepared, 5: completely prepared) for responding to a normal/COVID medical emergency / cardiac arrest. ${ }^{* *} \mathrm{p}<0.001$. The 'Count' axis is aligned such that response 3 ('Somewhat prepared (eg still unsure on aspects)') is equally distributed either side of 0 .

updated guidance on PPE, aerosol-generating procedures (AGPs) and adult life support $(91 \%, 84 \%$ and $91 \%$ respectively). In addition, $80 \%(n=45)$ of participants were aware that chest compressions are an AGP (as per local trust guidelines).

The following questions were assessed on a five-point Likert scale (1: low, 5: high). Participants felt significantly less prepared for a MET call in a patient with suspected COVID-19 than in a patient in whom it is not suspected; the mean Likert response was 2.4 and 3.9 respectively $(p<0.001$, Fig 1$)$. The same was true for cardiac arrest calls, with a mean response for patients with suspected COVID-19 of 2.2 compared with 3.9 for non-COVID-19 patients $(p<0.001$, Fig 1).

Prior to the simulation, participants were least confident in 'Acting as first responder in an emergency call to a suspected COVID-19 patient' (pre-simulation mean Likert score of 2.82) and in 'Managing the scenario if the patient passes away' (pre-simulation mean Likert score of 2.64). Table 1 outlines the scores for the remainder of the aspects of the emergency response assessed in Question 8 of the pre-simulation questionnaire.

All participants, including the observer, felt that the simulations improved their preparedness for responding to an emergency call in a patient with suspected COVID-19. This was evidenced by the fact that the mean Likert score for responding to COVID-19 MET calls increased from 2.4 to 3.7 ( $p<0.001$, Fig 2 ) after simulation. Similarly, the simulations increased the mean Likert score from 2.2 to 3.6 for COVID-19 cardiac arrest calls ( $p<0.001$, Fig 2$)$.

The simulations were run successfully. It was felt that the low-fidelity simulations were realistic enough to identify the challenges posed by an emergency in a patient with suspected COVID-19. This was evidenced by the fact that participants reported no concerns with the operating of the simulation apart from the fact that there was little briefing before the simulation. This was a design feature of the simulation, as one of the aims was to stress-test the current systems.

For further simulations, the participants responded with two main requests. The first was to run more simulations (of the same type) on a regular basis to improve skills. The second was 
Table 1. Confidence in undertaking different roles in the emergency response

\begin{tabular}{|c|c|c|c|}
\hline \multirow{2}{*}{$\begin{array}{l}\text { Aspect of the emergency } \\
\text { response }\end{array}$} & \multicolumn{2}{|c|}{ Mean Likert score } & \multirow[t]{2}{*}{ p-value } \\
\hline & Pre- & Post & \\
\hline $\begin{array}{l}\text { Putting out the appropriate } \\
\text { emergency call }\end{array}$ & 4.01 & 4.30 & $0.02^{*}$ \\
\hline $\begin{array}{l}\text { Acting as first responder in an } \\
\text { emergency call to a suspected } \\
\text { COVID-19 patient }\end{array}$ & 2.82 & 3.95 & $<0.001^{* *}$ \\
\hline $\begin{array}{l}\text { How to proceed during the } \\
\text { MET/arrest call }\end{array}$ & 3.11 & 4.00 & $<0.001^{* *}$ \\
\hline $\begin{array}{l}\text { Knowing what to do if a MET } \\
\text { call converts into an arrest call }\end{array}$ & 3.23 & 4.16 & $<0.001^{* *}$ \\
\hline $\begin{array}{l}\text { Knowing how to run/label } \\
\text { bloods/ABGs during an } \\
\text { emergency call }\end{array}$ & 3.21 & 3.86 & $<0.001^{* *}$ \\
\hline Donning and doffing PPE & 3.11 & 3.93 & $<0.001^{* *}$ \\
\hline $\begin{array}{l}\text { Managing the scenario if the } \\
\text { patient passes away }\end{array}$ & 2.64 & 3.14 & $<0.001^{* *}$ \\
\hline
\end{tabular}

The mean Likert score of participants' responses when asked to choose the most appropriate statement to 'I feel confident...' in the pre- and postsimulation questionnaires is shown.

${ }^{*} \mathrm{p}<0.05,{ }^{* *} \mathrm{p}<0.001$. ABG $=$ arterial blood gases

to run simulations that involved different skillsets (eg setting up (PAP).

\section{Assessment of the current emergency response}

The content of the faculty-led debrief and post-simulation questionnaire identified four key themes: communication, equipment, personnel and procedures. Table 2 details the issues and their potential solutions.
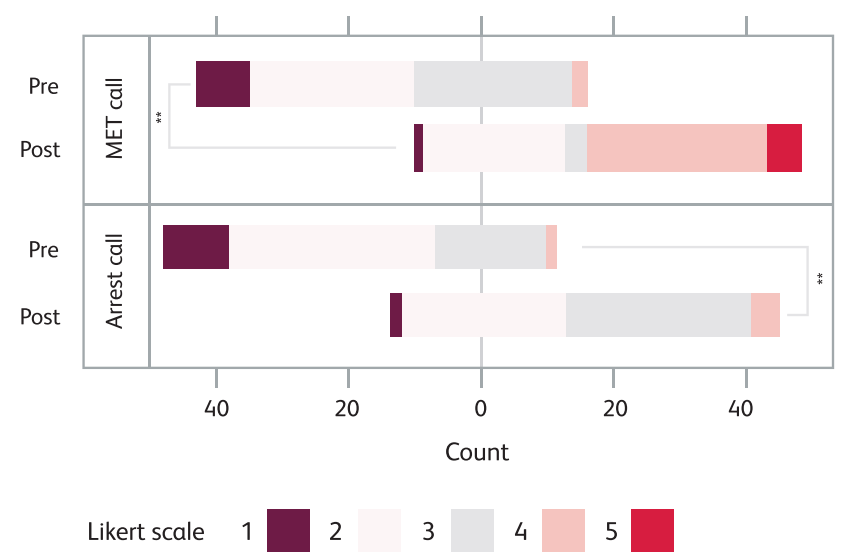

Fig 2. The responses on a Likert scale (1: completely unprepared, 5: completely prepared) for responding to COVID MET/arrest calls pre- and post-simulation. ${ }^{* * * *} p<0.001$. The 'Count' axis is aligned such that response 3 ('Somewhat prepared (eg still unsure on aspects)') is equally distributed either side of 0 .

\section{Discussion}

COVID-19 poses an unprecedented challenge to healthcare systems and workers globally. ${ }^{5}$ Healthcare professionals report a significant degree of unfamiliarity with the novel protocols and guidance, in particular the use of PPE and responding to MET calls. This study set out to demonstrate the potential role that low-fidelity simulation can play in supporting staff to feel more prepared to respond to challenging situations involving patients with suspected COVID-19.

At baseline, the participants felt significantly less prepared to deal with an emergency call in a patient with suspected COVID-19 than in a patient in whom COVID-19 is not suspected. After just two simulated scenarios involving patients with COVID-19, the participants felt significantly more prepared to respond to a medical emergency. In addition, participants felt significantly more confident to perform all aspects of the emergency response after a simulation.

The participants reported that the simulations were well run and without any significant operational issues. This is important as it demonstrates that low-fidelity simulations can be organised and identify issues with current guidelines in a timely manner. The simulations revealed issues in four key themes: communication, equipment, personnel and procedures.

In particular, the participants reported concerns over the feasibility of running a cardiac arrest with only four people inside the room (as was local NHS trust guidance on 4 April 2020), given that the following roles need to be shared among them: compressions, defibrillation (often linked to timing), procedures (eg $A B G$ and cannulation), team leader, airway management and in-room runner. In particular, the 'in-room runner' was a newly identified role and therefore it was felt that a minimum of five people were required to be inside the room (with some appropriate rotation of roles). Similarly, it was found to be crucial that there be a 'gatekeeper' outside the room, in addition to outside 'runners'. Table 2 outlines the issues raised and details potential solutions. A number of the solutions are relatively simple (eg producing 'grab bags' and equipment lists), further illustrating that low-fidelity simulation can aid in the development of quick, high-yield solutions. These solutions were specific to this NHS trust, and it is likely that performing simulations in different NHS trusts would identify different challenges and thus require different solutions.

This study has several limitations that require addressing. We describe the results of a small number of simulation groups, and as a result we are limited by the sample size available to us. Secondly, the simulation is set up with a COVID patient isolated in a side room, as this was the case for the majority of COVID patients at the conception of the study. However, this is unlikely to remain the case, with bays of COVID patients beginning to exist.

Additionally, this study only demonstrates an improvement in the participants' self-evaluated preparedness to respond to a COVID emergency and its different aspects. It is likely that this increased preparedness relates to an improved knowledge base and awareness of the skills required. However, we have not attempted to demonstrate improvement in those measures objectively.

Despite this, our methodology achieved the study aims of improving participants' preparedness to respond to emergency calls, supporting the notion that low-fidelity simulation is a useful tool in this context, and identifying the current gaps in the emergency response. This paper is an interim review as the 
Table 2. Descriptions of the identified issues and proposed solutions for responding to a medical emergency or cardiac arrest call for a patient with suspected COVID-19

\section{Issue}

\section{Communication}

1. Distance between the door and the patient led to further communication issues, participants who had relevant information (eg from documentation or imaging) outside the room felt unable to effectively communicate that information as they were not heard clearly or at times not acknowledged.

2. Given the fact that the notes must remain outside the side room in the 'green zone', it was reported that the door was a significant barrier to communicating information clearly.

3. The FFP3 masks make it more difficult to communicate clearly, with numerous participants reporting that they found it challenging to understand instructions.

4. It was noted to be important that any calls placed be specific, ie to include 'COVID-19' and 'male/female', also to clarify whether it was a MET call or cardiac arrest call, and what PPE was required on arrival of the MET team.

\section{Equipment}

1. The notes and drug chart were often taken into the room ('red zone') and were therefore contaminated. This obviously provides significant challenges to any further management of this patient. If the notes are left outside the room, this limits their usefulness and requires one individual to read the notes from outside, and possibly requires an individual close to the door on the inside to receive the information.

2. There were numerous concerns about what equipment could be taken into the room. Examples included computers, resuscitation trolleys, emergency medication and defibrillators.

3. Challenges arose in transferring the essential equipment for investigation and resuscitation (eg ABGs) in a timely fashion and avoiding having to doff and re-don. There was also an absence of bags to safely hand over 'red' ABG bottles to 'green' individuals outside the room.

4. It was often found that the candidate's first instinct was to enter the room once donned without identifying any equipment needs in the room (eg defibrillator). Once they entered the room, they were unable to exit to grab any equipment and they had to rely on the team outside, which led to delays.

5. It was difficult to safely dispose of sharps as there were no sharps bins in the room. There were no bins in the side room / outside the room for doffing of kit.

6. It was reported that nursing staff are being advised that the MET team will arrive with their own PPE (confirmed source from an actual MET call), despite no MET members being made aware of this or having the provision to do so.
Solution

1. To help overcome the significant delays in being able to access the notes, we propose that a 'red' summary sheet for each query COVID-19 patient should remain inside the 'red zone', as a copy of a version from the full notes. This sheet could include current diagnosis, current plan, significant past medical history, allergies, current treatment, escalation plan and can be used as a proforma in the notes to exclude duplicating work.

2. We advise that on arrival to an emergency for a query COVID19 patient, the team announce their arrival to the in-room team, assess what equipment they currently have inside, what equipment they require and how many people are currently in the room.

3. We would like to stress the fact that clear closed-loop communication will be essential in these stressful situations, and to encourage the use of names and confirmations.

4. This can be achieved through training and education of staff.

1. See Communication solution 1 for discussion of the COVID proforma which may aid the decision-making of the in-room team.

2. A list of items can be placed on each resuscitation trolley to identify which items can be taken into the side room and which cannot.

3. 'Transfer bags' for the safe transfer of samples should be stored on the top of the resuscitation trolley.

4. Creating a 'grab bag', containing useful items for a MET call (eg $A B G$, cannula, blood bottles, gauze, tourniquets), to be stored on the resuscitation trolley or outside all side rooms containing patients with possible or confirmed COVID-19. Any individual entering the room should ask the in-room team what equipment they have and what else they require. This individual should bring in that equipment at that point to avoid having to doff and re-don.

5. Provide the appropriate kit inside for doffing and disposal of equipment within 'red' areas.

6. Ensure the same information is communicated to all teams. 
Table 2. Descriptions of the identified issues and proposed solutions for responding to a medical emergency or cardiac arrest call for a patient with suspected COVID-19 (Continued)

\section{Issue}

\section{Procedures}

1. Transfer of any material from inside the room to outside (eg sending of an $A B G$ ) was found to be complicated. In both situations, an $A B G$ was handed from the 'red' team to the 'green' individual outside the room. In both cases, this resulted in potential contamination of the individual and other members of staff.

2. In scenario two, the initial MET call converts into an arrest call, requiring the complete doffing and re-donning of PPE to ensure that all members of the team were in AGP PPE before starting chest compressions. The delay as a result of doing this was $\sim 4$ minutes (therefore without chest compressions).

Staff reported a lack of confidence and preparation in donning and doffing safely, as was evidenced by the fact that many participants contaminated themselves.

3. The nurse who had been part of the simulation when non-AGP PPE was required had not passed his fit test, which led to a replacement needing to be found as the scenario proceeded to a cardiac arrest; this increased delays.

4. There was much uncertainty as to which $A B G$ machine the blood from a patient with suspected COVID-19 could be run on.

5. There was also uncertainty as to when the MET/arrest team had arrived as they began to start donning and this led to anxiety among the clinical staff inside the room.

\section{Personnel}

1. Given that there were significant communication challenges due to a combination of masks, distance and barriers, one individual was often required to hover close to the door / peel away from the arrest to relay information out of the room or receive any relevant information.

2. In both simulations there was occasionally no one immediately outside the room, and therefore no access to notes, imaging, drug chart, medications or any other equipment. In both simulations, a participant ended up doffing and standing outside the room to support.

3. When any investigations needed to be run (eg $A B G$ ), the person outside the room in both simulations became unavailable, meaning that there was no one to relay information to the 'red' team.

4. Not all staff attending arrest calls have been fit tested.
Solution

1. We recommend that in order to safely send investigations, the following protocol should be applied. A 'red' individual should label the bottle and put the cap on it in the room. They should then communicate with the 'gatekeeper' or 'runner' to ask them to open a 'transfer bag'. Using non-touch technique (both individuals), the ABG bottle should be dropped into the 'transfer bag' before being sealed by the 'green' team. The 'runner' should then take the ABG to the nearest appropriate machine and run it without touching the inside of the bag. It is imperative that bloods are labelled with stickers while in the 'red' zone.

2. Provision of training for staff on donning and doffing.

3. Provision of adequate fit testing and awareness of a team's fit-test status.

4. Education of staff and dissemination of information.

5. MET team to announce their presence on arrival by knocking and also to begin identifying equipment and personnel needs (as per Equipment solution 4).

1. Our simulation highlighted the fact that it may be challenging and unsafe to run a cardiac arrest call with only four individuals in the room. One member of the team needs to be relatively free inside the room to relay information from outside the room (eg documentation, imaging) to the team. Then, among the remaining three members of the team, the following roles need to be fulfilled: chest compressions, airway management (eg bag-valve mask), defibrillation, timing, procedures (eg ABGs and cannulation) and one member leading the team.

2. Additionally, it is imperative that there is a 'gatekeeper' outside the room at all times to coordinate the flow of people and equipment into the room and to communicate information from the notes or previous investigations. This person should not leave their post under any circumstances, as doing so would isolate the in-room team from further support.

3. We also advise that there is an additional runner outside the room to collect any further equipment or send any investigations.

4. We recommend that there be a list of staff on every ward who is on shift and has passed fit testing for rapid identification if additional support is required. 
results of further simulations, including scenarios in different environments, will be published in a later article. Low-fidelity in situ simulation is dependent upon the availability of space and staff, which, although present now, may not persist. As such, the authors made the decision to publish an interim report as it felt necessary to disseminate the initial conclusions swiftly in order to support other NHS trusts.

The current climate demonstrates the need for clear, concise and effective guidance. This simulation study has acted as a stress test for current NHS trust protocols.

This study provides clear evidence that low-fidelity clinical simulation can be organised quickly, with minimal resources, and identify issues with current guidance while also providing the opportunity to develop workable solutions. In addition, we received very positive feedback that it improved the preparedness of the staff who will be involved in emergency calls to patients with suspected COVID-19. We recommend that NHS trusts nationwide adopt this approach in light of the COVID-19 pandemic.

\section{Acknowledgements}

We would like to thank the participants of the simulations.

\section{References}

1 Datta R, Upadhyay KK, Jaideep CN. Simulation and its role in medical education. Med J Armed Forces India 2012;68:167-72.
2 La Cerra C, Dante A, Caponnetto V et al. Effects of high-fidelity simulation based on life-threatening clinical condition scenarios on learning outcomes of undergraduate and postgraduate nursing students: A systematic review and meta-analysis. BMJ Open 2019;9:e025306.

3 Lewis R, Strachan A, Smith MM. Is high fidelity simulation the most effective method for the development of non-technical skills in nursing? A review of the current evidence. Open Nurs $]$ 2012;6:82-9.

4 Massoth C, Röder H, Ohlenburg H et al. High-fidelity is not superior to low-fidelity simulation but leads to overconfidence in medical students. BMC Med Educ 2019;19:29.

5 Walker PGT, Whittaker C, Watson $\mathrm{O}$ et al. The global impact of COVID-19 and strategies for mitigation and suppression. London, 26 March 2020: Imperial College COVID-19 Response Team. www. imperial.ac.uk/media/imperial-college/medicine/sph/ide/gida-fellowships/Imperial-College-COVID19-Global-Impact-26-03-2020v2.pdf [Accessed 5 May 2020].

6 Li L, Lin M, Wang X, Bao P, Li Y. Preparing and responding to 2019 novel coronavirus with simulation and technology-enhanced learning for healthcare professionals: challenges and opportunities in China. BMJ Simul Technol Enhanc Learn 2020:bmjstel-2020-000609.

Address for correspondence: Dr Rhys Wenlock, Brighton and Sussex University Hospitals NHS Trust, Eastern Road, Brighton BN2 5BE.

Email: r.wenlock@nhs.net 